\title{
Advantages of applying the mixture when guniting on vertical surfaces of building envelope
}

\author{
Anatoliy Abramenko, Elena Gorbaneva*, Aleksey Semyenov, Andrey Mishchenko, Dmitriy \\ Loboda \\ Voronezh State Technical University", Moscovskiy prospect, 14, Voronezh, 394026, Russia
}

\begin{abstract}
Having studied the technological modes of applying the mixture by the guniting method on hard unyielding surfaces, we can conclude that the technology for applying building mixtures is formed depending on changes in a number of factors (including the strength characteristics and the rebound of the mixture from the vertical surface). Experimental study of the influence of the technological parameters of application on the efficiency of guniting with the classical method and application using a nozzle for vortex application allows us to visually detect the zones of rational application of this technology. Based on the experimental data, we carried out an analysis of the influence of the technological parameters of applying the mixture on the criterion indicators. For this, we investigated various methods $[8,9]$, processed the results and revealed dependencies, discovered unknown aspects of gunning with the use of a nozzle for vortex application of a construction mixture. The assessment showed a sufficient degree of coincidence of theoretical and experimental studies and the possibility of their practical application, on the basis of which we can conclude about the efficiency of the nozzle for the vortex application of the building mixture.
\end{abstract}

\section{Introduction}

The use of classical nozzles for gunning does not completely solve the problem of sticking and subsequent clogging of the nozzle with the mixture. Adjustment of the nozzle operation consists in varying the mixing zone of the cement and water, as well as changing the opening of the sprayer, which is carried out most often manually when starting the device into operation. The consequence of such mixing is almost inevitably the adhesion of the mixture inside the nozzle and the subsequent need to stop the process, washing and restart the system in operation. This procedure is quite time consuming, and if the operator does not have sufficient experience, such a malfunction can occur every 1-2 hours and significantly reduce the efficiency and productivity of the entire complex.

*Corresponding author: egorbaneva@,vgasu.vrn.ru 
Having studied the technological modes of applying the mixture by the method of guniting on hard unyielding surfaces, we can conclude that the technology for applying building mixtures is formed depending on changes in a number of factors [1-3]:

- the distance from the nozzle to the gunited surface with an interval of $0.5-1.5 \mathrm{~m}\left(\mathrm{~h}_{\mathrm{opt}}\right.$ $=1.0 \mathrm{~m}$ ),

- the composition of the granules, which is selected so that the fractions in the sprayed mass have equal proportions (if this parameter is maintained, it is possible to keep a minimum rebound).

The formula for finding the ultimate strength of concrete:

$$
R_{\text {con }}=k R_{c} \cdot(C / W-0.43)
$$

where $k$ - the coefficient taking into account the influence of the method of applying the concrete mixture. For guniting $k=0.38-0.43$;

$\mathrm{R}_{\mathrm{c}}$ - cement activity.

It should be noted that in this formula only the amount and mass of the mixture are taken into account, this is not enough for a more accurate determination of the limit.

The strength of the concrete depends on the speed of application, the performance of the guniting machine $\mathrm{Q}$, the distance to the application surface $\mathrm{h}$, and the method of application $\mathrm{S}$ (classic and with a nozzle for vortexing the mixture). Thus, it is necessary to introduce correction factors into this ratio, which would take into account the technological parameters of guniting. This will allow the most reliable characterization of the influence of the main parameters on the strength properties of the mixture, applied by the classical method and by using a nozzle for vortex application of the mixture.

\section{Materials and methods}

To determine the most suitable composition of the gunite mixture, let us set the watercement ratio $(\mathrm{W} / \mathrm{C})$ and granulometry with constant values, taking into account practical recommendations and experiments.

As a result of the inevitable difference in the distribution of the components of the cement-sand mortar, in the number of defects (pores, microcracks) and the variability of the properties of individual grains of the mixture and cement paste, material heterogeneity appears, which affects the test results. Achieving anisotropy, that is, the directionality of the structure and, accordingly, increased strength is one of the main properties of a cementsand mortar. The properties of the sprayed mass differ to some extent from the properties of concrete obtained by the standard method, which means that anisotropy will be achieved with other parameters of the mortar.

Since when planning an experiment, it is most difficult to choose the correct interval for changing parameters, we take as a basis the same variations as when conducting studies of applying a mixture onto hard unyielding surfaces. Determination of technological parameters $\mathrm{Q}, \mathrm{h}, \mathrm{S}$ should be carried out on the basis of the analysis of previously conducted gunite experiments.

Let us assign the distance of application in the range of $0.5-1.5 \mathrm{~m}$, and the speed of the concrete mixture flow, that is, the productivity $\mathrm{Q}-5-9 \mathrm{~m}^{3} / \mathrm{h}$.

Thus, we have intervals of variation of the following technological parameters:

$\mathrm{Q} \in[5 ; 9]$

$\mathrm{h} \in[0.5 \mathrm{~m} ; 0.75 \mathrm{~m} ; 1 \mathrm{~m} ; 1.5 \mathrm{~m} ; 1.5 \mathrm{~m}]$;

$\mathrm{S} \in[\mathrm{S} 1$ (classic method);

S2 (with a vortex nozzle)]. 
Having considered the obtained values of the distances of application and the method of application at different productivity, we draw a plan of experimental work.

In laboratory conditions, we built the installation, shown in Figures 1 and 2.

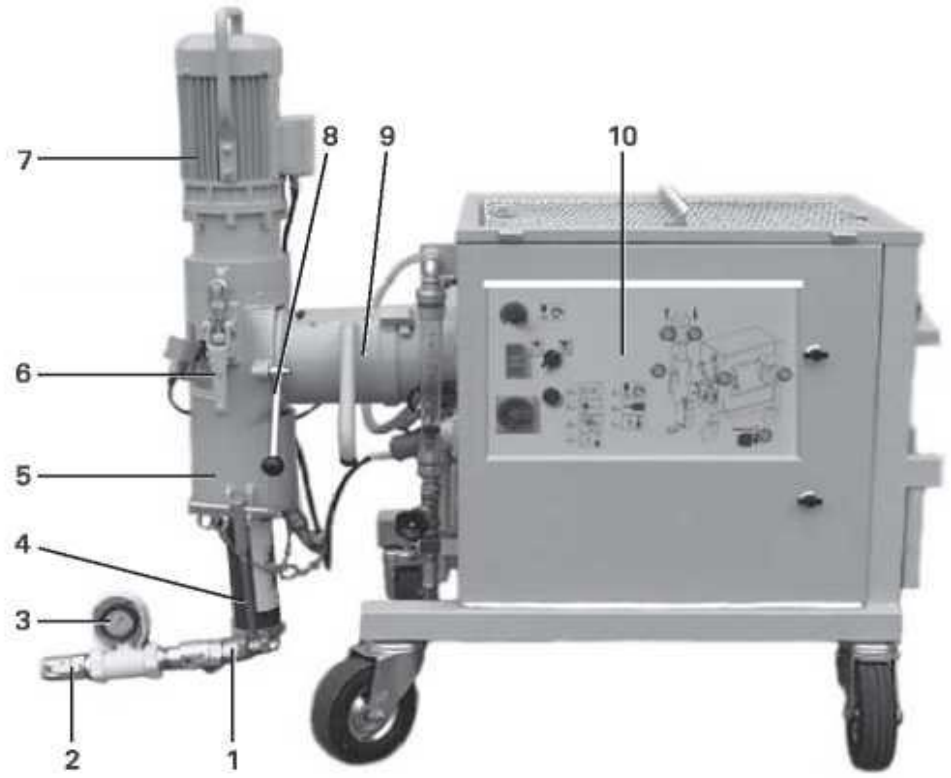

Fig. 1. Pump duo-mix 2000 mixer. Left side view. [4]

1 - the pump tip. 2 - the sleeve for mortar incl. M35. 3 - the sleeve pressure gauge. 4 - the screw pump (stator incl. rotor). 5 - the mixing pump pipe. 6 - the fixation for the pump drive. 7 - the pump motor. 8 - the fixation for the mixing pump pipe. 9 - the mixer pipe. 10 - the control panel.

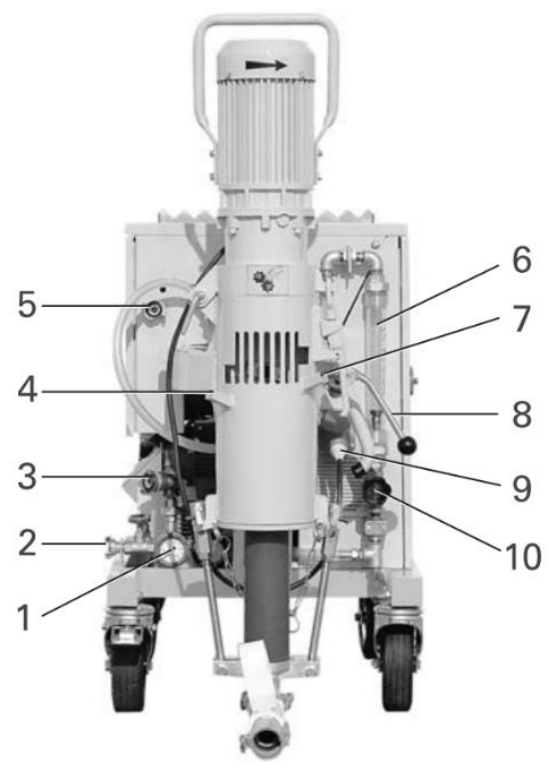

Fig. 2. Pump duo-mix 2000 mixer. Front view. [4]

1- the inlet water pressure gauge. 2 - the cleaning tap. 3 - the GEKA-coupling for connecting the pump for water inlet. 4 - fixing device 1 for the raw material level sensor. 5 - the air hose connection 
(spraying). 6 - the flow meter. 7 - mounting device 2 for the raw material level sensor. 8 - the fixing arm for the mixer pipe. 9 - the input plug for the pump motor. 10 - the valve for fine flow control

As standard, the duo-mix 2000 mixing pump (fig. 1) is used for feeding from bags. With the help of additional equipment, it is possible to fill with a transfer hood or filter hood from a silo or with a mixing tube with a funnel for pasty material [4].

Mixing and pumping devices have different drives. The mixing area consists of two chambers. In the first chamber, dry material is combined with water and mixed. In the second chamber, the mixed material undergoes a mixing stage. This helps to obtain a finished product of uniform consistency and high quality. The ready-to-use material is dispensed by a screw pump. Water is supplied directly to the mixing chamber and can be monitored by a water supply sensor. If the water supply pressure is unacceptably high, the water pressure sensor automatically turns off the device. If the water supply pressure is low, the water pump continues to operate.

A raw material level sensor checks the fill level of the pump mixing tube. The mixer motor and water pump are automatically turned off and go into standby mode when the highest level is reached. The mixer motor and water pump are automatically switched on again if the level sensor detects the minimum fill level. This prevents the mixer from overfilling. It is also optionally possible to install a dry material level sensor in the material tank. If it signals a lack of material, the mixer drive, water pump and pump drive are automatically turned off. Once the material has entered the tank again, an error indicator appears on the two-stage button Ein/Aus. The machine can then be turned on again by pressing the Ein/Aus button.

The machine is equipped with a frequency converter, which makes it possible to smoothly select the number of rotations of the pump motor.

The entire machine can be controlled (i.e. Ein/Aus on and off (On/Off):

- directly using buttons in the control cabinet;

- when using a spray device with an air supply valve located on it;

- when using a spraying device using the switch located on it at the point of its connection.

Guided by the adopted plan for the interval of changing technological parameters, we applied concrete using a vortex nozzle (Fig. 3). At the same time, in the process of guniting the mixture onto the prepared surface, we obtained all the necessary samples for testing the concrete for strength [5-7].

The initial setting period of the mortar is approximately 2-3 hours. Further, according to the regulations of GOST, we cut out 6-12 samples - cubes with an edge of $2 \mathrm{~cm}$ of the gunite mass with a metal plate. After that, it is necessary to determine the values of the compressive strength and enter the corresponding coefficients.

Before testing, all concrete samples are checked, the detected deposits are removed, small cavities are filled with cement paste. It should be noted that the strength index determined during the tests depends both on the properties of the material and on the method by which the tests are carried out. In practice, it seems impossible to obtain samples of concrete that are identical in structure.

All test results are generated in the test laboratory video report. 


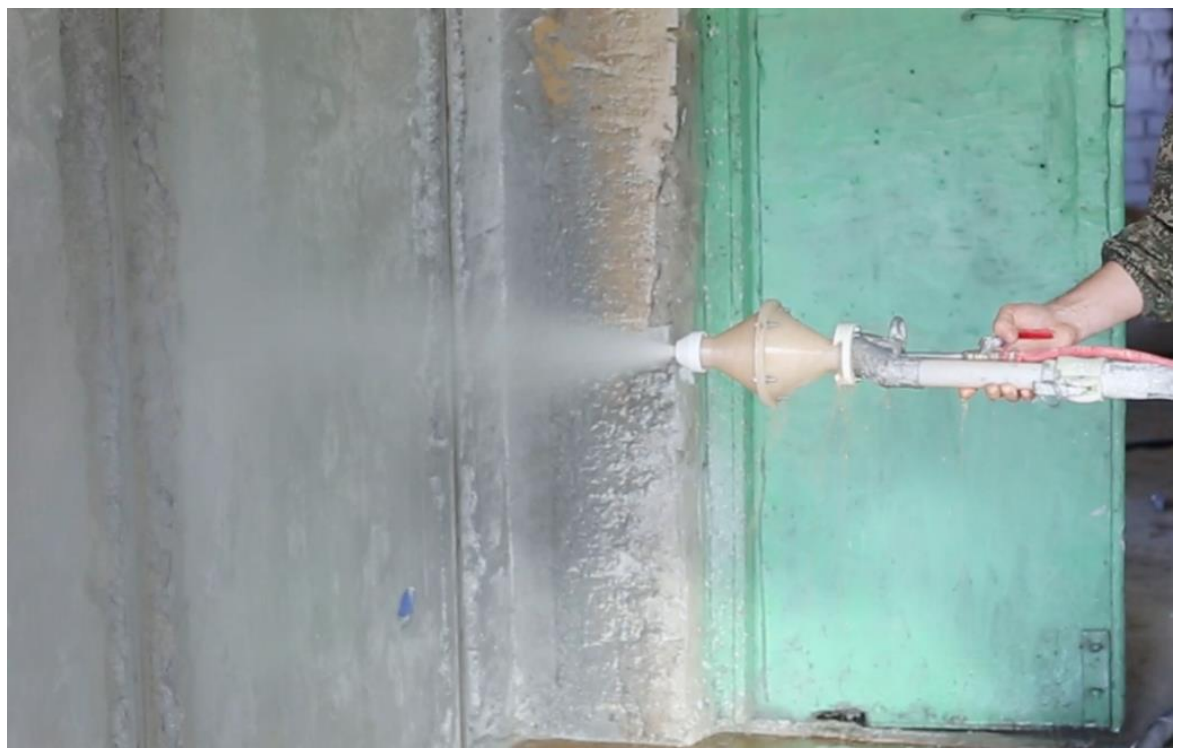

Fig.3. Application of the mortar using a nozzle for swirling the mixture onto a vertical surface

One of the main problems in guniting is the rebound effect of the particles. In order to minimize rebound losses, we proposed a method for applying a mortar in such a way that, due to the creation of a swirl of the supplied mass, an additional vector of force arises that prevents it.

To assess the effectiveness of the method, it is necessary to carry out an experimental comparison of the methods of applying the mortar with other technological parameters being equal. In addition to the parameter of the minimum rebound and the specified strength of concrete during guniting, it is necessary to take into account the factor of the peeling off of the applied mixture. This is due to the fact that the thicker the layer of the sprayed mass applied, the stronger the effect of gravity, and the weaker the sticking forces. Based on the experimental values of the dimensions of the concreting spots, we calculate the thickness of the layer that is laid per unit of time. Let us take $t=1 \mathrm{~s}$, the speed of movement of the nozzle in the process of guniting $-30 \mathrm{~cm} / \mathrm{s}$.

Taking into account the performance of the mix duo 2000 plastering station and the thickness of the applied layer, we find the critical distance of application, that is, the maximum possible thickness of the sprayed mass layer held on the surface in one pass.

The size of the concreting spot is determined by the formula (2):

$$
D_{\text {spot }}=D_{j}+30 \mathrm{~cm} / \mathrm{s} \times t
$$

where $D_{j}-$ the gunite jet diameter;

$\mathrm{D}_{\text {spot }}$ - the concreting spot size;

$30 \mathrm{~cm} / \mathrm{s}$ - the nozzle travel speed;

$\mathrm{t}$ - the nozzle travel time, equal to $1 \mathrm{~s}$.

Thus, the layer thickness $T_{1}$ is determined by formula (3):

$$
T_{l}=\frac{Q \times t}{3600 F_{\text {spot }}}
$$

where Q - the experiment installation performance;

$\mathrm{F}_{\text {spot }}$ - the concreting spot area;

$\mathrm{t}$ - the application time. 
Next, it is necessary to determine the boundaries of the "rebound-free" zone of variation of the technological parameters of gunite and compare the data depending on the method of application.

\section{Results}

Let us conduct an experiment based on a two-factor model on a three-level matrix using a classic nozzle (Table 1) and a nozzle for vortex application of the mixture (Table 2). We find the dependence of the strength of the mixture on the distance of application $\left(\mathrm{x}_{1}\right)$, and the method of application $\left(\mathrm{x}_{2}\right)$. Experiments should be carried out to prove that the amount of rebound will be minimal when using a vortex nozzle.

The initial data of the experiment were the speed and method of application. Based on them, it is necessary to find the boundaries of the "rebound-free" zone of variation of the application parameters.

Since when planning an experiment, it is most difficult to choose the correct interval for changing parameters, we take as a basis the same variations as when conducting studies of applying a mixture to hard unyielding surfaces.

Determination of technological parameters Q, h, S should be carried out on the basis of the analysis of previously conducted experiments on guniting.

Let us assign the distance of application in the range of $0.5-1.5 \mathrm{~m}$, and the speed of delivery of the concrete mixture, that is, the productivity $\mathrm{Q}-5-9 \mathrm{~m}^{3} / \mathrm{h}$.

Thus, we have intervals of variation of the following technological parameters:

$\mathrm{Q} \in[5 ; 9]$;

$\mathrm{h} \in[0.5 \mathrm{M} ; 0.75 \mathrm{M} ; 1 \mathrm{M} ; 1.5 \mathrm{~m} ; 1.5 \mathrm{M}]$;

$\mathrm{S} \in[\mathrm{S} 1$ (classic method);

S2 (with a vortex nozzle)].

We considered the obtained values of the distances of application and the method of application at different productivity and then draw a plan of experimental work.

Table 1. The obtained values of rebound and strength of the finished surface with varying the distance of the spray pattern and the productivity of the installation with a classic method

\begin{tabular}{|l|c|c|c|c|}
\hline No & $\begin{array}{c}\text { Spray distance, } \mathbf{m} \\
\left(\mathbf{x}_{\mathbf{1}}\right)\end{array}$ & $\begin{array}{c}\text { Installation } \\
\text { productivity } \mathbf{~ m}^{\mathbf{3}} / \mathbf{h} \\
\left(\mathbf{x}_{\mathbf{2}}\right)\end{array}$ & Rebound, \% & $\begin{array}{c}\text { Surface strength, } \\
\mathbf{~ m P a}\end{array}$ \\
\hline 1 & 0.5 & 2.5 & 26 & 16.5 \\
\hline 2 & 0.75 & 2.5 & 18 & 15.1 \\
\hline 3 & 1 & 2.5 & 9 & 14.0 \\
\hline 4 & 1.25 & 2.5 & 0 & 12.5 \\
\hline 5 & 1.5 & 2.5 & 0 & 12.0 \\
\hline 6 & 0.5 & 5 & 31 & 15.0 \\
\hline 7 & 0.75 & 5 & 22 & 14.8 \\
\hline 8 & 1.0 & 5 & 12 & 13.9 \\
\hline 9 & 1.25 & 5 & 5 & 12.9 \\
\hline 10 & 1.5 & 5 & 0 & 12.0 \\
\hline 11 & 0.5 & 7.5 & 39 & 14.5 \\
\hline 12 & 0.75 & 7.5 & 27 & 13.0 \\
\hline 13 & 1.0 & 7.5 & 15 & 12.0 \\
\hline 14 & 1.25 & 7.5 & 6 & 11.5 \\
\hline 15 & 1.5 & 7.5 & 2 & \\
\hline
\end{tabular}


Table 2. The obtained values of the rebound and the strength of the finished surface with varying the distance of the spray pattern and the productivity of the installation by the nozzle for vortex application of the mixture

\begin{tabular}{|l|c|c|c|c|}
\hline No & $\begin{array}{c}\text { Spray distance, } \mathbf{m} \\
\left(\mathbf{x}_{\mathbf{1}}\right)\end{array}$ & $\begin{array}{c}\text { Installation } \\
\text { productivity } \mathbf{~ m}^{\mathbf{3}} / \mathbf{h} \\
\left(\mathbf{x}_{\mathbf{2}}\right)\end{array}$ & Rebound, \% & $\begin{array}{c}\text { Surface } \\
\text { strength, } \mathbf{~ m P a}\end{array}$ \\
\hline 1 & 0.5 & 2.5 & 8 & 16.8 \\
\hline 2 & 0.75 & 2.5 & 0 & 14.7 \\
\hline 3 & 1 & 2.5 & 0 & 13.5 \\
\hline 4 & 1.25 & 2.5 & 0 & 12.1 \\
\hline 5 & 1.5 & 2.5 & 0 & 11.5 \\
\hline 6 & 0.5 & 5 & 12 & 16.5 \\
\hline 7 & 0.75 & 5 & 2 & 16.1 \\
\hline 8 & 1.0 & 5 & 0 & 15.9 \\
\hline 9 & 1.25 & 5 & 0 & 15.5 \\
\hline 10 & 1.5 & 5 & 0 & 15.4 \\
\hline 11 & 0.5 & 7.5 & 13 & 165 \\
\hline 12 & 0.75 & 7.5 & 6 & 16.2 \\
\hline 13 & 1.0 & 7.5 & 1 & 15.9 \\
\hline 14 & 1.25 & 7.5 & 0 & 15.7 \\
\hline 15 & 1.5 & 7.5 & 0 & 15.4 \\
\hline
\end{tabular}

The results of experiments showing the influence of technological parameters on the efficiency of guniting in the classical method and when applied using a vortex nozzle are shown in the diagrams. The analysis of the constructed surfaces allows us to see the areas of rational application of this technology.

In Figures 4-7, we present the changes in the degree of rebound and strength depending on the distance of the spray pattern and the productivity of the guniting machine for different application methods with the same other parameters.

After analyzing the experimental part of the surfaces and comparing them with the theoretically calculated one, it should be concluded that the theoretical and experimental data are quite close; the discrepancy between the rebound values at the border of the theoretical "rebound-free" zone was 7.2\%.

Surface strength, $\mathrm{mPa}$

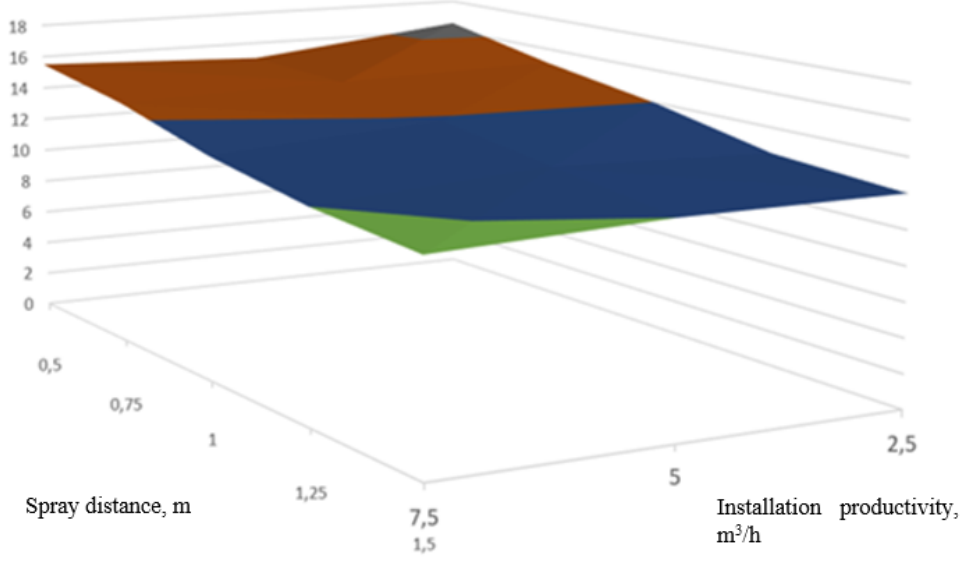

Fig.4. Dependence of the surface strength on the distance of the spray and the productivity of the installation when using a classic nozzle 
Surface strength, $\mathrm{mPa}$

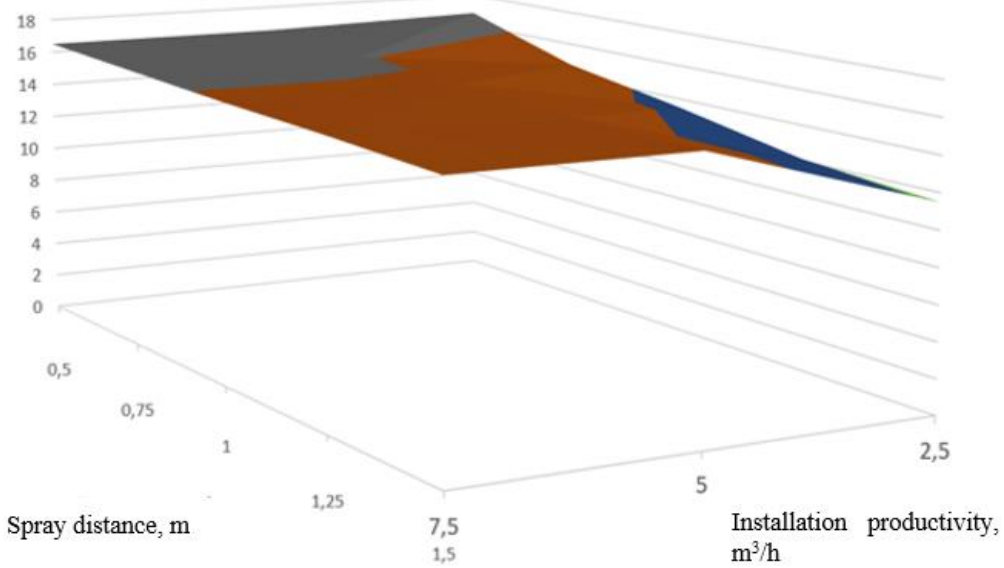

Fig.5. Dependence of the surface strength on the distance of the spray and the productivity of the installation when using a nozzle for vortex application of the mixture

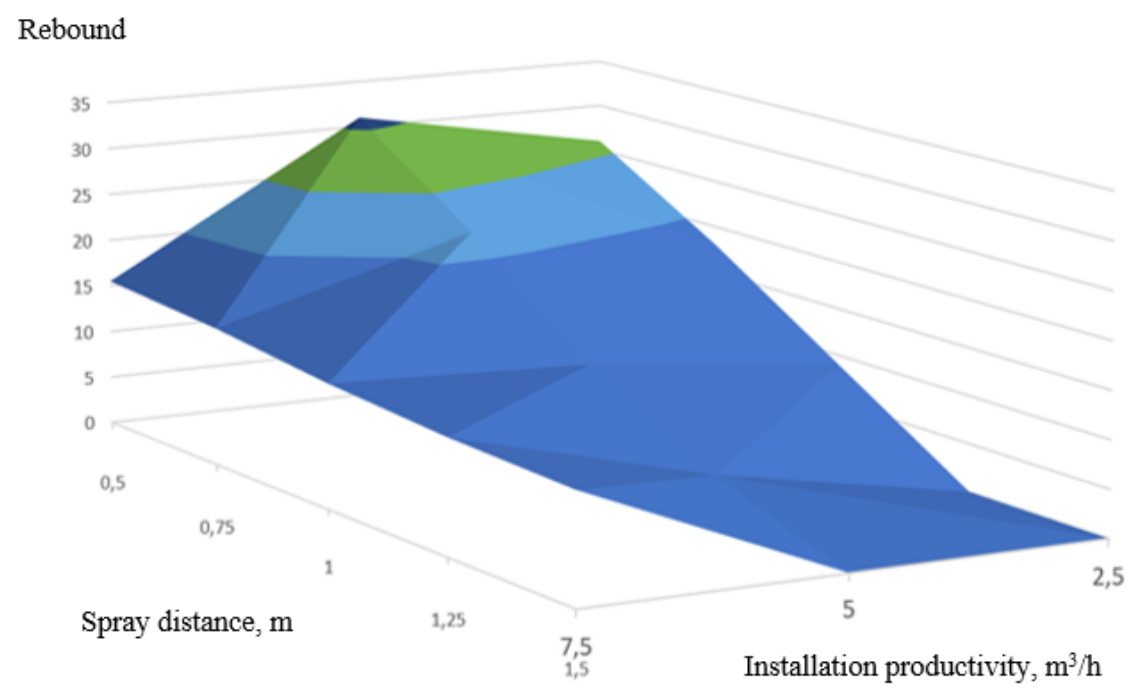

Fig.6 Dependence of the rebound of particles on the distance of the spray and the productivity of the installation when using a classic nozzle 


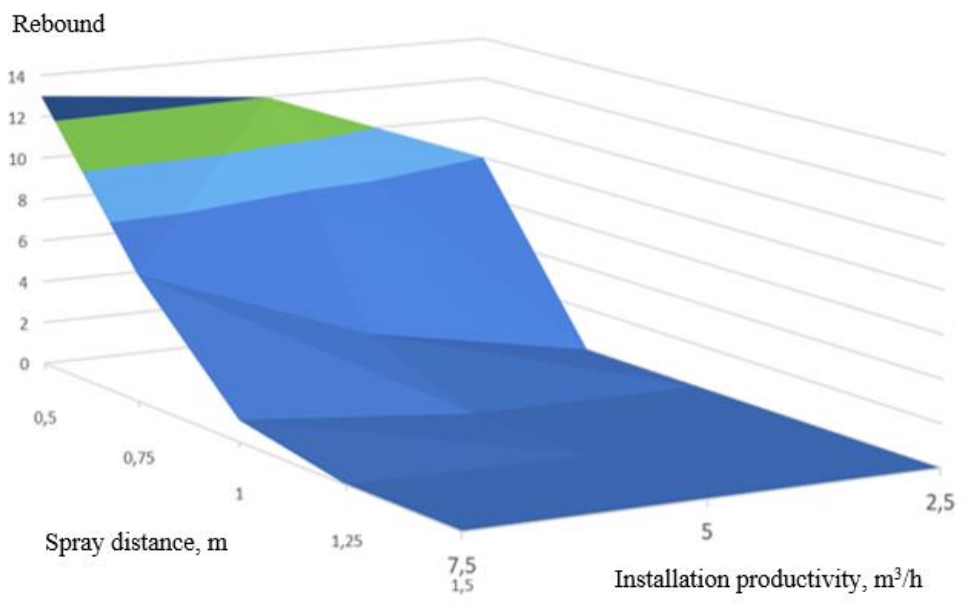

Fig.7. Dependence of the rebound of particles on the distance of the spray and the productivity of the installation when using a nozzle for vortex application of the mixture

\section{Conclusions}

Thus, the assessment of the influence of technological parameters on criterion indicators, such as strength characteristics and rebound of the mixture from a vertical surface, showed a sufficient degree of coincidence between theoretical and experimental data. This makes it possible to apply in practice the method of guniting by vortex application. In addition, the following conclusions can be drawn about the efficiency of the vortex nozzle:

- the range of the non-rebound zone has significantly expanded (from $0.75 \mathrm{~m}$ to $1.5 \mathrm{~m}$ ) in all performance intervals;

- the dispersion of the application has improved organoleptically;

- the strength of concrete slightly increased (by $8 \%$ ), which can be explained by the adhesion of particles at a higher application rate, respectively, better compaction, which is better observed with a decrease in the spraying distance of the mixture;

- at the extreme values of productivity and distance, the surface strength during the vortex method of the application did not change, which indicates a more significant decrease in the translational vector of particle motion and determines the boundaries of the parameters for using a vortex nozzle, which can be taken into account when determining the operating procedure.

\section{References}

1. A.A. Saus, V.V. Panchenko, V.V. Oplachko, Concrete gunning in modern construction. Varieties, advantages, scope. Achievements in science and education. (2019)

2. Li, Pengcheng \& Zhou, Zhi \& Chen, Lianjun \& Liu, Guoming \& Xiao, Wei. Research on Dust Suppression Technology of Shotcrete Based on New Spray Equipment and Process Optimization, Advances in Civil Engineering, 11 (2019) doi.org/10.1155/2019/4831215.

3. J. Samuel, \& K. Denis, Comparison between thin spray-on liners and shotcrete as surface support mechanisms in tunnels, IJIRAE: International Journal of Innovative 
Research in Advanced Engineering V, 329-337 doi://10.26562/IJIRAE.2018.OCAE10084.

4. Duo-mix 2000 mixing pump. Operating instructions. 386685 - 2006-06. 37p (2006). https://iam-almaz.ru/files/public/manuals/m-tec/m-tec-duo-mix-200-manual.pdf

5. P.N. Sukhorebryi, Determination of the flow structure in the spiral chamber of a radialaxial reversible hydraulic machine based on numerical simulation of the fluid flow, Mechanical engineering problems, 13(1), 31-41 (2010)

6. V.Ya. Mishchenko, E.P. Gorbaneva, E.V. Ovchinnikova, K.S. Sevryukova, Improving the energy efficiency of residential buildings during major repairs, FES: Finance. Economy. Strategy 16(1) 66-72 (2019)

7. V.Ya. Mishchenko, E.P. Gorbaneva, A.A. Abramenko, Faizi Ahmad Reshad New technologies in the construction industry, In the collection: Organization of construction production. Materials of the II All-Russian Conference. From 15-26 (2020)

8. R. Temam, Navier - Stokes Equations. Theory and numerical analysis (2nd ed. Mir. Moscow, 1981)

9. V.Ya. Mishchenko, E. Gorbaneva, et al., Planning the Optimal Sequence for the Inclusion of Energy-Saving Measures in the Process of Overhauling the Housing Stock, Advances in Intelligent Systems and Computing 983, 79-91 (2019) doi:10.1007/978-3-030-19868-8_8

10. S.A. Kolodyazhnyy, V.Ya. Mishchenko et al., The influence of design features of housing facilities on energy consumption, E3S Web of Conferences 175, 11019 (2020) doi:10.1051/e3sconf/202017511019 